\title{
Evaluación de la disponibilidad, utilización y costos de la tomografía computarizada en el estado de Morelos, México
}

\author{
John Pérez-Rodríguez,MC, ${ }^{(1)}$ Juan Luis Gerardo Durán-A renas, MC, PhD, ${ }^{(2)}$ \\ Sendy Isarel Hernández-Gaytán, IQ , M en $C,{ }^{(3)}$ Laura Leticia Tirado-Gómez, MC, M en $C$, ${ }^{(4)}$ \\ Francisco Javier Díaz-Vásquez, MC, ${ }^{\left({ }^{5}\right)}$ Malaquías López-C ervantes, MC, PhD. ${ }^{(5)}$
}

\begin{abstract}
Pérez-Rodríguez J, Durán-Arenas JLG, Hernández-Gaytán SI,Tirado-Gómez LL, Díaz-Vásquez FJ, López-Cervantes M. Evaluación de la disponibilidad, utilización y costos de la tomografía computarizada en el estado de Morelos.

Salud Publica Mex 2002;44:392-398.
\end{abstract} El texto completo en inglés de este artículo está disponible en: http://www.insp.mx/salud/index.html

\section{Resumen}

Objetivo. Evaluar la disponibilidad, utilización y costos económicos de la tomografía computarizada (TC) en hospitales públicos y privados en el estado de Morelos, México. Material y métodos Mediante un estudio transversal efectuado entre enero y abril de 1999, se evaluaron cuatro hospitales en el estado de Morelos, dos públicos y dos privados. Se llevaron a cabo entrevistas individuales con los directores, administrado res, médicos radiólogos y jefes de mantenimiento. Se realizó un análisis estadístico descriptivo de las variables, disponibilidad, utilización y costos, asi como una evaluación integral de la tomografía, haciendo énfasis en la variabilidad de los patrones observados en los hospitales participantes en el estudio. Resultados. LaTC es una tecnología utilizada en los hospitales del estado de Morelos desde hace más de 10 años. Aun cuando existen programas de mantenimiento preventivo y correctivo a los equipos de tomografía, sus costos son muy elevados. Además se carece de estrategias que permitan evaluar la tecnología antes de su adquisición y durante su utilización. El estudio no incluyó aspectos relativos a la correcta indicación clínica de la TC, ni a sus posibles efectos secundarios. Conclusiones. Los hallazgos obtenidos a partir de la presente in-
Pérez-Rodríguez J, Durán-Arenas JLG, Hernández-Gaytán SI,Tirado-Gómez LL, Díaz-Vásquez FJ, López-Cervantes M. Assessment of the availability, utilization, and costs of computerized tomography in Morelos, Mexico.

Salud Publica Mex 2002;44:392-398.

The English version of this paper

is available at: http://www.insp. mx/salud/index.html

\section{A bstract}

Objective.To assess the availability, utilization, and costs of computerized tomography (CT scan) in private and public hospitals in Morelos State, Mexico. Material and Methods. From January to A pril 1999, a cross-sectional study was carried out in two private and two public hospitals in Morelos, Mexico. Individual face-to-face interviews were conducted with directors, managers, radio logists, and heads of maintenance at each hospital. Statistical analysis was performed to describe the variables measuring availability, utilization, and costs of CT scans.A comprehensive assessment of CT scans was also performed. Emphasis was made on the variability of obser ved patterns among the participating hospitals. Results. CT scan technology has been used by hospitals in Morelos State for over ten years; programs for preventive or corrective maintenance of these equipments are available, although at high costs. No strategies for technology assessment are available for acquisition of CT scanners nor during their period of utilization. This study did not attempt to evaluate the appropriateness of the clinical use of CT nor its untoward effects. Conclusions Findings from the present study showed that: 1) a lack of mechanisms for technology assessment and management of CT

John Pérez Rodríguez agradece muy especialmente al Consejo $\mathrm{N}$ acional de Ciencia y Tecnología por la beca recibida durante el posgrado.

(1) Sistema Estatal para el Desarrollo Integral de la Familia,Veracruz, México.

(2) Instituto Mexicano del Seguro Social. México, D.F., México.

(3) Dirección General de Salud A mbiental. Secretaría de Salud. México, D.F., México.

(4) Instituto $\mathrm{N}$ acional de Cancerología. Secretaría de Salud. México, D.F., México.

(5) Coordinación General de los Institutos N acionales de Salud. Secretaría de Salud. México, D.F., México.

Fecha de recibido: 22 de febrero de 2001 - Fecha de aprobado: 3 de mayo de 2002

Solicitud de sobretiros: Dr. Malaquías López-Cervantes. Coordinación General de los Institutos N acionales de Salud. Periférico Sur 4118, Primer Piso. Colonia Jardines del Pedregal, 01900. México, D. F.

Correo electrónico: mlopez@ mail.ssa.gob.mx 
vestigación fueron los siguientes: a) existe una carencia de procesos de evaluación y gestión tecnológica, que permita a las unidades hospitalarias un manejo eficiente de la tomografía computarizada, y b) también se carece de mecanismos para regular la adquisición, evaluar la tecnología y seleccionar las mejores alternativas en función de su efectividad, eficacia, seguridad y accesibilidad. El texto completo en inglés de este artículo está disponible en: http:// www.insp.mx/salud/index.html

Palabras clave: to mo grafía computarizada por rayos $X$; evaluación de tecnología biomédica; costos; disponibilidad; México scans prevented hospitals from managing $\mathrm{CT}$ scanning technologies efficiently; 2) technology assessment regulation is not available, even though it is necessary for the adequate selection of the best technologies, on the basis of their efficiency, effectiveness, safety, and availability. The English version of this paper is available at: http://www.insp.mx/salud/ index.html

Key words: tomography, X-Ray computed; technology assessment, biomedical; costs; availability; M exico a evaluación de la disponibilidad y utilización de L tecnología médica es un campo poco desarrollado en México. A pesar de ello existen estudios en los que se han explorado conceptualmente, y en la práctica, algunos de los aspectos del campo de la evaluación de tecnología. Entre estos estudios encontramos la encuesta de disponibilidad y utilización de nueva tecnología que se llevó a cabo en 1983, ${ }^{1}$ cuyo objetivo fue evaluar 17 tecnologías que en ese momento constituían la punta de lanza de la innovación en salud. Este estudio mostró, en primer lugar, la existencia de concentración de los recursos en las grandes ciudades (Distrito Federal, Guadalajara y Monterrey); por otra parte, mostró que la decisión para la adquisición de nuevas tecnologías era tomada en el ámbito central y ésta se realizaba sin atender a los aspectos relacionados con su costo-efectividad. Otro hallazgo de importancia fue el hecho de que algunas de las tecnologías de alto costo permanecían almacenadas por mucho tiempo, antes de ser instaladas; esto debido a la falta de infraestructura necesaria o la falta de recursos humanos capacitados para su operación.

El estudio destaca la necesidad de generar criterios claros que permitan regular la adquisición, la adopción y difusión de la tecnología en el contexto de una práctica médica madura, lista para incorporar y aprovechar plenamente las nuevas tecnologías. ${ }^{1}$

En el mismo sentido, pero analizando la concentración que se da en la producción de equipos, encontramos las publicaciones de Cruz, Sánchez EC, ${ }^{2-4}$ Martuscelli y Faba. ${ }^{4,5}$ Por otra parte Frenk ${ }^{6,7}$ y Lastiri $\mathrm{QS}^{8}$ se concentran en el desarrollo conceptual de la evaluación de tecnología y en la necesidad de elaborar inventarios de disponibilidad y utilización de las tecnologías en México.

En cuanto a la regulación de la tecnología para la salud, es importante mencionar que en México no existe una instancia reguladora central que se dedi- que específicamente a la evaluación de la tecnología para la salud, ${ }^{9,10}$ existiendo, por tanto, desconocimiento de cuál es el impacto real de muchas de ellas. No obstante, en algunas instituciones públicas de servicios de salud se han realizado diversos intentos para dar seguimiento a la tecnología para la salud, mediante la formación de comités de vigilancia; a pesar de ello, no se han consolidado o desarrollado de una manera completa. ${ }^{9}$

Actualmente el campo de la tecnología de la salud en México sigue enfrentando problemas similares a los reportados hace 20 años, como la importación de tecnologías médicas de altos costos sin una evaluación previa y adecuada, debido tanto a la falta de un sistema de evaluación de tecnología y de una estructura institucional que opere dicho sistema, como a la falta de un sistema de información que permita la retroalimentación sobre los usos apropiados de las tecnologías médicas recién introducidas. ${ }^{4,11}$

De acuerdo con lo antes mencionado, la evaluación de la tecnología médica debería formar parte de los procesos normativos y de planificación de todo sistema de salud. ${ }^{12}$ La investigación que aquí se presenta es un ejemplo de evaluación de una tecnología médica de alto costo, la tomografía computarizada, gracias a los siguientes indicadores: disponibilidad, mantenimiento, operación y costo por servicio otorgado. Situación que nos permitió conocer la utilización de esta tecnología en el estado de Morelos, así como los principales problemas encontrados en su operación.

\section{Material y métodos}

Se evaluó la disponibilidad, utilización y costos de la tomografía computarizada en hospitales públicos y privados con más de 30 camas censables en el estado de Morelos, durante los meses de enero a abril de 1999. El diseño del estudio fue de tipo transversal y se uti- 
lizó el método de encuestas dirigidas al personal de los cuatro hospitales seleccionados.

Como primera instancia para la obtención de información se hizo un censo telefónico en todos los hospitales del estado de Morelos. Este censo identificó a cuatro hospitales que cuentan con tomografía computarizada: dos privados y dos públicos. El personal de los cuatro hospitales aceptó participar en la investigación, para lo cual se efectuaron entrevistas individuales dirigidas a los directores, administradores, cuatro médicos radiólogos encargados de la operación de la tomografía computarizada y cuatro jefes de mantenimiento. El tiempo requerido para cada una de las entrevistas fue de 90 minutos en promedio. Las entrevistas se realizaron durante la jornada laboral.

El cuestionario se dividió en dos secciones: la primera, dirigida al director del hospital, y al administrador, incluía 17 preguntas relacionadas con adquisición, utilización y costos de adquisición. La segunda sección, dirigida a los jefes de imagenología y de mantenimiento, incluyó 61 preguntas relacionadas con utilización, disponibilidad y mantenimiento.

Dentro del análisis se llevó a cabo, en una primera fase, un análisis estadístico descriptivo de las siguientes variables dependientes: disponibilidad, utilización, costos de operación; variables independientes: mantenimiento, operación, tipo de hospital, complejidad de la atención al paciente, recursos materiales y financieros.

Posteriormente se utilizó la información recabada para desarrollar una comparación integral de la disponibilidad y utilización de la tomografía computarizada, mediante la medición de las variaciones en los patrones de utilización, tanto en hospitales públicos como en los hospitales privados. Finalmente se hizo un análisis comparativo de las variaciones de patrones de utilización entre ambos tipos de hospitales.

En relación con disponibilidad se valora la presencia de la tomografía computarizada en los hospitales y su capacidad para producir servicios. Se recabó la información mediante entrevistas al personal directivo, médico y administrativo sobre el equipo, incluyendo modelo, año de fabricación, año de compra, fecha de inicio de uso y capacidad de servicio.

En el caso de utilización, se consideró el uso clínico de la tomografía computarizada en los hospitales para lo cual se entrevistó al personal médico con el fin de conocer los motivos o razones de la indicación del uso de la tecnología, el diagnóstico previo al uso de ésta, número de estudios necesarios para realizar el diagnóstico, así como el número de estudios por paciente.

El costo de operación fue definido como los costos regulares que se utilizan para financiar los pro- gramas y la prestación de servicios año tras año. La información se obtuvo por medio de la entrevista al personal directivo y administrativo. De esta manera se obtuvo el costo de adquisición de la tomografía computarizada, su antigüedad, los costos de mantenimiento y de los insumos utilizados en su operación. Con esta información se calcularon los costos por servicios producidos con la tomografía computarizada.

Mediante la revisión de la bitácora de operación se contabilizó el número de servicios producidos en 24 horas, tipo de servicio, insumos y recursos humanos utilizados y el costo por servicio producido.

Para la evaluación del mantenimiento de la tomografía se revisó el diagnóstico situacional de los hospitales y las condiciones en las que se encontraba dicha tecnología, recabándose además información sobre la existencia de contrato de mantenimiento, periodicidad del mismo, tipo de mantenimiento (preventivo y correctivo), costos y garantía del equipo.

Para este estudio se utilizaron además las siguientes definiciones:

Tomografía computarizada: técnica en la cual una computadora reconstruye los datos tomados de una revisión del cuerpo del paciente efectuada con rayos $X$ desde todos los ángulos, en un solo plano, cuyo objetivo es el de obtener imágenes diagnósticas transversales al plano del paciente. ${ }^{13}$

Evaluación de tecnología médica: forma integral de investigar las consecuencias técnicas (casi siempre clínicas) y sociales del empleo de las tecnologías médicas, tanto a corto como a largo plazo, así como sus efectos directos e indirectos, deseados y no deseados. ${ }^{14}$

Hospital público: hospital que pertenece al Estado y recibe presupuesto estatal y federal.

Hospital privado: hospital que funciona con recursos de la iniciativa privada.

\section{Resultados}

En cuanto al tipo de tecnología disponible en los hospitales, se encontró que los hospitales públicos (No.1 y No.2) tienen un tomógrafo de origen japonés, de marca Shimadzu TCT 450 TE, y un tomógrafo de origen alemán, de marca Siemens Somatom CR, respectivamente. Los hospitales privados (No. 3 y No. 4) tienen un tomógrafo de origen alemán, de marca Pecimens Somatom CR, y un tomógrafo de origen Japonés, de marca Toshiba TS 300, respectivamente.

El cuadro I muestra la disponibilidad de la tomografía. Se observa que los hospitales tienen un promedio de 12 años de haber sido inaugurados, y el hospital No.3 es el de mayor antigüedad (20 años). Entre los 
Cuadro I

Disponibilidad de la tomografía en cuatro hospitales del estado de Morelos, México, 1999

\begin{tabular}{|c|c|c|c|c|}
\hline \multirow[b]{3}{*}{$\begin{array}{l}\text { Condiciones de la tecnología en el mo- } \\
\text { mento de la compra }\end{array}$} & \multicolumn{2}{|c|}{ Hospitales públicos } & \multicolumn{2}{|c|}{ Hospitales privados } \\
\hline & No.1 & \multirow[t]{2}{*}{$\mathrm{N} 0.2$} & \multirow[t]{2}{*}{ No.3 } & \multirow[t]{2}{*}{$\mathrm{N} 0.4$} \\
\hline & N ueva & & & \\
\hline Antigüedad en el hospital & 14 años & 12 años & 20 años & 5 años \\
\hline $\begin{array}{l}\text { Motivos reportados en la falta de funcio- } \\
\text { nalidad del tomógrafo }\end{array}$ & $\begin{array}{l}\text { - Falta de refacciones en } \\
\text { el país porque son de } \\
\text { importación } \\
\text { - Problemas administra- } \\
\text { tivos (burocráticos) } \\
\text { - Problemas econó- } \\
\text { micos }\end{array}$ & $\begin{array}{l}\text { - Falta de refacciones en } \\
\text { el país se tardan apro- } \\
\text { ximadamente de } 1 \text { a } 3 \\
\text { meses importarlo por } \\
\text { problemas aduanales y } \\
\text { arancelarios } \\
\text { - Fallas de energía eléc- } \\
\text { trica }\end{array}$ & $\begin{array}{l}\text { - Falta de software } \\
\text { - Falla del tubo } \\
\text { - Fallas eléctricas }\end{array}$ & $\begin{array}{l}\text { - Falta de refacciones en } \\
\text { el país }\end{array}$ \\
\hline Generación del tomógrafo & Cuarta generación & Segunda generación & Segunda generación & Segunda generación \\
\hline Evaluación para el cambio de equipo. & No & $\begin{array}{l}\text { Sí una tomografía de 3ra } \\
\text { generación }\end{array}$ & No & No \\
\hline $\begin{array}{l}\text { Evaluación de la tomografía en alguna } \\
\text { ocasión }\end{array}$ & No & No & No & No \\
\hline Fecha de recepción del tomógrafo. & Febrero de 1994 & Enero de 1990 & N oviembre de 1990 & Diciembre de 1994 \\
\hline $\begin{array}{l}\text { Fecha de inicio de utilización del to- } \\
\text { mógrafo }\end{array}$ & Septiembre de 1995 & Febrero de 1991 & Febrero de 1991 & Diciembre de 1994 \\
\hline
\end{tabular}

Fuente: Encuesta realizada en el estado de Morelos en 1999

problemas del funcionamiento de la tomografía se mencionan en los cuatro hospitales la falta de refacciones en el país y, con menor frecuencia, problemas económicos y administrativos. En los cuatro hospitales no se realizó un análisis o evaluación de la tecnología previo a su adquisición.

En relación con la fecha de recepción y la de utilización, el hospital No.2 fue el que reportó la instalación más reciente (cuatro meses), y el hospital No.1 la más antigua (19 meses); situación debida a la presencia de infraestructura inadecuada, o la falta de personal médico y técnico. Un hallazgo importante fue el hecho de que algunos equipos iniciaron su operación con posterioridad al vencimiento de la garantía proporcionada por el proveedor, provocando la pérdida de la misma y elevando los costos de mantenimiento y reparaciones cuando éstas fueron requeridas.

En el cuadro II se presentan los costos de la tomografía; es importante notar que los costos varían considerablemente entre los hospitales públicos y privados. El hospital público No.2 presenta el costo más alto de mantenimiento (\$35 126). Asimismo, este hospital presenta los costos más altos por insumos utili- zados (\$36 800). Sin embargo, el costo promedio más alto para el paciente por la utilización de la tomografía corresponde a los hospitales privados, en particular el hospital No.4 con 2100.00 pesos, y el costo más bajo corresponde al hospital público No.1 con 45700 pesos.

El cuadro III presenta la persona responsable de la solicitud del estudio; los médicos especialistas predominaron en tres de los cuatro hospitales. Entre los diagnósticos más frecuentes que motivan la solicitud del estudio predominan el traumatismo craneoencefálico y los tumores; mientras que los diagnósticos más frecuentemente establecidos después del uso fueron los hematomas y el infarto cerebral.

Se reportó que bastaba con un estudio para establecer un diagnóstico, y entre las complicaciones más frecuentes durante el uso de la tomografía se mencionó la reacción a los medios de contraste.

En cuanto al mantenimiento de la tomografía, en el cuadro IV se muestra que los cuatro hospitales tienen la garantía caducada; el hospital privado No.4 no tiene ni contrato ni programa de mantenimiento preventivo, esto debido a los altos costos del servicio. Por 


\section{Costos de la tomografía en cuatro hospitales del estado de Morelos, México, en 1999}

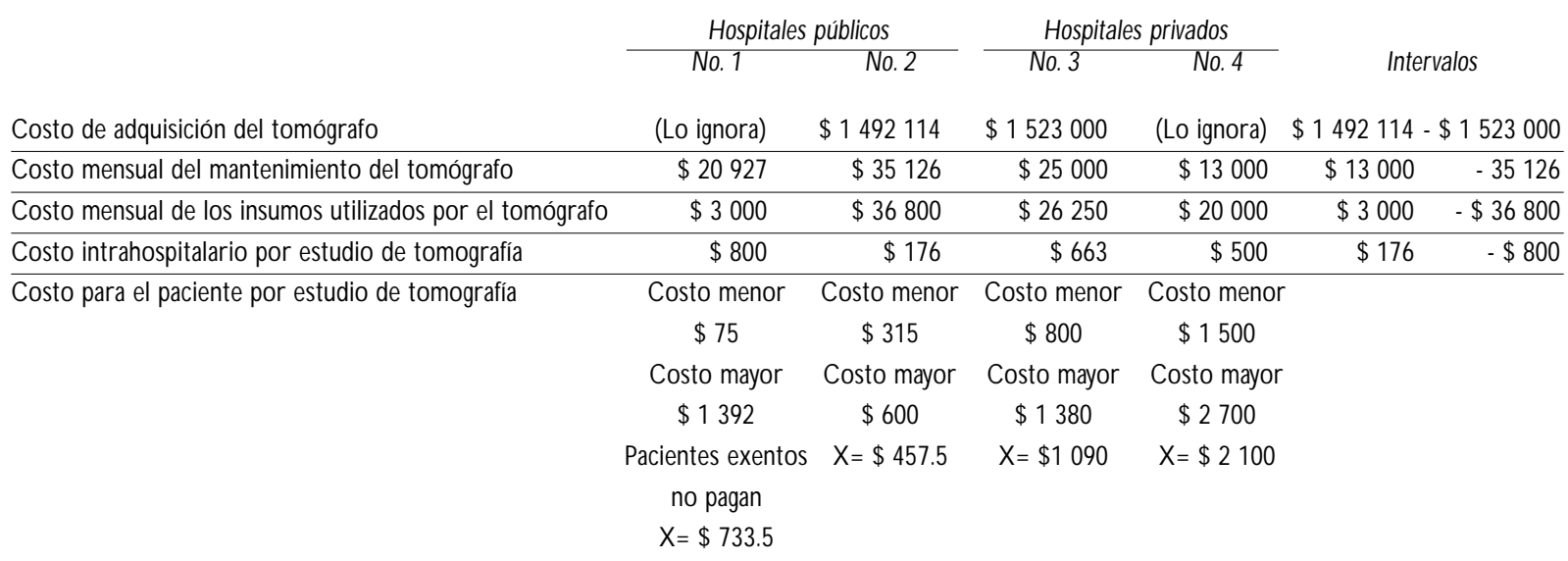

Fuente: Encuesta realizada en el estado de Morelos en 1999

\section{Cuadro III}

Uso de la tomografía en cuatro hospitales del estado de Morelos, México, en 1999

\begin{tabular}{|c|c|c|c|c|}
\hline \multirow[b]{3}{*}{$\begin{array}{l}\text { Responsable de la solicitud } \\
\text { del estudio }\end{array}$} & \multicolumn{2}{|c|}{ Hospitales públicos } & \multicolumn{2}{|c|}{ Hospitales privados } \\
\hline & No.1 & No.2 & No.3 & No.4 \\
\hline & $\begin{array}{l}\text { Médico general, médicos es- } \\
\text { pecialistas, médicos parti- } \\
\text { culares }\end{array}$ & Médicos especialistas & Médicos especialistas & Médicos especialistas \\
\hline $\begin{array}{l}\text { Diagnósticos más frecuentes } \\
\text { previos al uso de la tomo- } \\
\text { grafía }\end{array}$ & $\begin{array}{l}\text { - Traumatismos cráneo } \\
\text { encefálicos } \\
\text { - Crisis convulsivas } \\
\text { - Cefalea }\end{array}$ & $\begin{array}{l}\text { - Traumatismos cráneo } \\
\text { encefálico } \\
\text { - Enfermedad vascular cere- } \\
\text { bral } \\
\text { - Procesos oncológicos }\end{array}$ & $\begin{array}{l}\text { - Tumores } \\
\text { - Cáncer } \\
\text { - Traumatismos cráneo } \\
\text { encefálicos }\end{array}$ & $\begin{array}{l}\text { - Traumatismos cráneo } \\
\text { encefálicos } \\
\text { - Enfermedad vascular } \\
\text { cerebral } \\
\text { - Procesos oncológicos }\end{array}$ \\
\hline $\begin{array}{l}\text { Diagnósticos más frecuentes } \\
\text { reportados como resultado } \\
\text { del uso de la tomografía }\end{array}$ & $\begin{array}{l}\text { - Traumatismos cráneo } \\
\text { encefálicos (hematomas y } \\
\text { contusiones) } \\
\text { - Cisticercosis } \\
\text { - Tumores }\end{array}$ & $\begin{array}{l}\text { - Hematomas } \\
\text { - Infartos } \\
\text { - N eurocisticercosis }\end{array}$ & $\begin{array}{l}\text { - Cisticercosis } \\
\text { - Infarto cerebral } \\
\text { - Tumores }\end{array}$ & $\begin{array}{l}\text { - Hematomas subdurales } \\
\text { - Infarto cerebral } \\
\text { - Confirmación de cáncer }\end{array}$ \\
\hline $\begin{array}{l}\text { Número de estudios para lle- } \\
\text { gar a un diagnóstico }\end{array}$ & 1 & 1 & 1 & 1 \\
\hline $\begin{array}{l}\text { Complicaciones más fre- } \\
\text { cuentes durante el uso de la } \\
\text { tomografía }\end{array}$ & $\begin{array}{l}\text { - Efectos indeseables cuando } \\
\text { utilizan medios de con- } \\
\text { traste endovenosos } \\
\text { - Rash } \\
\text { - Anafilaxia }\end{array}$ & $\begin{array}{l}\text { Reacción a los medios de } \\
\text { contraste }\end{array}$ & $\begin{array}{l}\text { Reacción a los medios de } \\
\text { contraste }\end{array}$ & $\begin{array}{l}\text { - Efectos colaterales se- } \\
\text { cundarios por los radio } \\
\text { fármacos } \\
\text { - Mareo, náusea, urticaria, } \\
\text { vómito } \\
\text { - Muerte }\end{array}$ \\
\hline
\end{tabular}

Fuente: Encuesta realizada en el estado de Morelos en 1999

otra parte, los cuatro hospitales informaron tener mantenimiento correctivo oportuno, aunque esto sucede en búsqueda de apoyo urgente, lo que resulta en un costo mayor del servicio.
La productividad de la tomografía también fue explorada. En la figura 1 se muestra que los hospitales públicos tienen mayor productividad: el hospital público No.1 tiene el promedio de estudios más alto en salud pública de méxico / vol.44, no.5, septiembre-octubre de 2002 


\section{Cuadro IV \\ Mantenimiento de la tomografía en cuatro hospitales del estado de Morelos, México, en 1999}

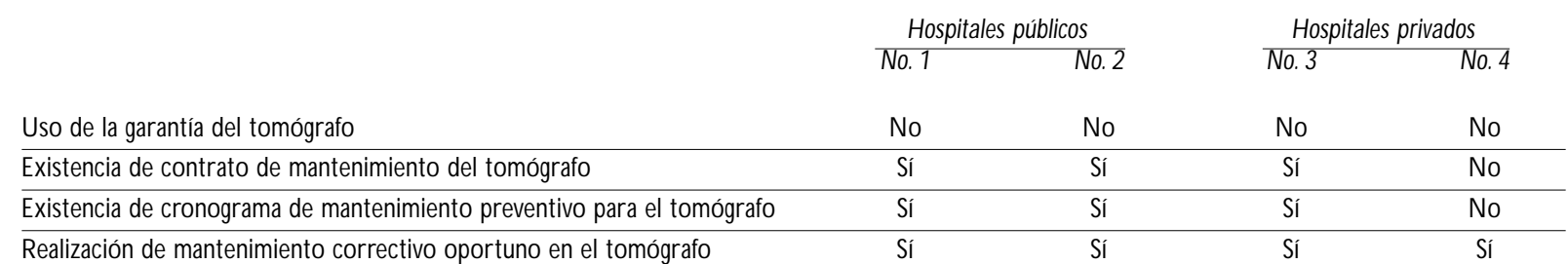

Fuente: Encuesta realizada en el estado de Morelos en 1999

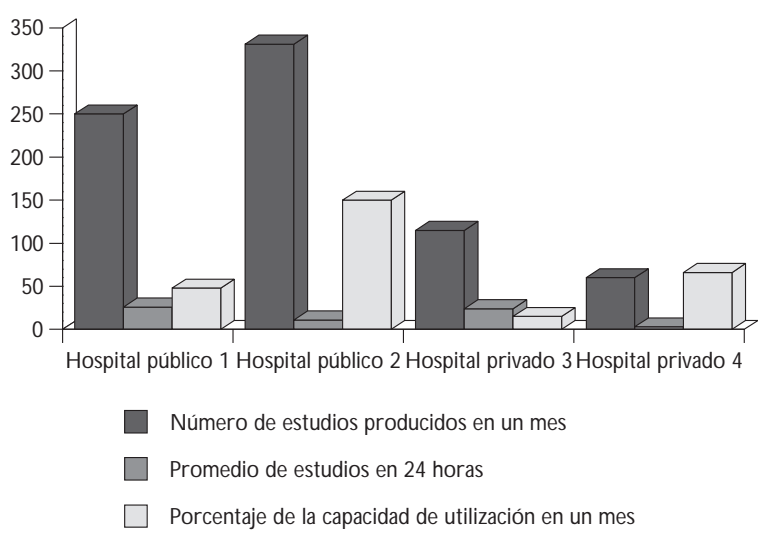

Figura 1. Productividad de la tomografía en cuatro hospitales del estado de Morelos, México, 1999

24 horas, con 26 y el hospital público No. 2 presenta el mayor número de estudios producidos en un mes, con 331. En contraparte, el hospital privado No.3 presenta el porcentaje más bajo de capacidad de utilización en un mes, con 15, y el hospital privado No.4 presenta el promedio más bajo de estudios en 24 horas, con tres.

\section{Discusión}

Aunque el estado de Morelos no se caracteriza por ser uno de alto desarrollo económico, llama la atención el incremento que ha tenido en la última década la importación de tecnología médica de alta complejidad. Los resultados de esta investigación muestran que los cuatro tomógrafos en todo el estado se localizan en la ciudad de Cuernavaca. Si consideramos que la población del estado es de 1442662 habitantes, y el municipio de Cuernavaca es el de mayor población, con
316782 habitantes, ${ }^{15}$ es claro que existe un exceso de este tipo de tecnología. En Canadá, en los años 80, el estándar era contar con un equipo de tomografía computarizada por cada millón de habitantes.

Es de notar que tanto en los hospitales públicos como en los privados existe una falta de evaluación y de gestión tecnológica que permitan un manejo eficiente de la tomografía computarizada. De hecho, uno de los hospitales públicos está pensando en sustituir su equipo por uno de tercera generación a pesar de que en el mercado ya se cuenta con equipos de quinta generación.

En el ámbito estatal no existe regulación para la evaluación de la tecnología médica, tampoco se valora la capacidad instalada, y mucho menos si la utilización de esta tecnología se da en condiciones adecuadas y con una óptima efectividad.

En el ámbito hospitalario sigue predominando la misma situación de hace casi 20 años hallada en otros trabajos publicados por nuestro equipo, ${ }^{1,16,17}$ como la falta de racionalización en la incorporación y uso de la tecnología. La falta de regulación para la incorporación de la tecnología médica se debe a las siguientes situaciones: espacios inadecuados para su instalación, falta de capacitación del personal médico y técnico para el manejo de la tecnología, arreglos inadecuados con las compañías que proporcionan mantenimiento preventivo y correctivo, situaciones que al final generan duplicidades y altos costos en la utilización de la tecnología.

No obstante lo anterior, es importante reconocer que el problema que debe encarar la evaluación de la tecnología no consiste simplemente en adoptar o rechazar una tecnología nueva y compleja en un marco de creciente preocupación por los costos, sino la selección, entre varias opciones, de las mejores tecnologías en función de su efectividad, eficiencia y la seguridad para el usuario. 


\section{A gradecimientos}

Este trabajo es producto de la investigación realizada para obtener el grado de Maestría en Administración de Servicios de Salud, en el Instituto Nacional de Salud Pública, de John Pérez-Rodríguez, quien agradece el apoyo de las autoridades del Instituto. A Nora Velia Ramírez por su colaboración en la elaboración del manuscrito.

\section{Referencias}

1. Rodríguez-D omínguez J, Vandale-Toney S, Durán-Arenas JL, López S, MCN ally AP. La disponibilidad de recursos tecnológicos en la medicina mexicana; resultados de una encuesta nacional. Salud Publica Mex 1984;26:336.

2. Gómez D 0, Cruz C, Nigenda G. La regulación participativa de la atención a la salud. México, D.F.: Fundación Mexicana para la Salud, 1994;29-32.

3. Sánchez EC ,A rredondo A, C ruz C . La investigación en tecnología médica: apoyo a la cooperación entre países. Salud Publica Mex 1991;33:513519.

4. Cruz C, Faba G, Martuscelli J. La tecnología de la salud en México. Salud Publica Mex 1992;34(supl):145-156.

5. Martuscelli-Q uintana J, Faba-Beaumont G. El desarrollo tecnológico en el marco del Programa Nacional de Salud. Salud Publica Mex 1988;30: 853-858.
6. Frenk J, Lozano R, González-Block MA, Ruelas E, Bobadilla JL, Zurita E. Economía y salud:propuesta para el avance del sistema de salud en México. Informe final. México, D.F.: Fundación Mexicana para la Salud, 1994.

7. Frenk J, Peña J. Bases para la evaluación de la tecnología y la calidad de la atención a la salud. Salud Publica Mex 1988;30:405-415.

8. Lastiri Q S. Hacia una estrategia nacional de fomento al uso racional de tecnologías en salud. México, D.F.: Fundación Mexicana para la Salud, 1993:1-7.

9. Durán-A renas JLG, López-Cervantes M,Tirado-Gómez L. Evaluación de tecnologías en salud 2000. México D.F.: Fundación Clínica Médica SurCON ACYT, 2001:81-93.

10. Granados A. La evaluación de las tecnologías médicas. Med Clin 1995;104:581-565

11. López-Cervantes M,Tirado-Gómez L, D e Icaza-del Río E, D urán-Arenas JLG . Las tecnologías médicas y la salud. ¿Mucho ruido y pocas nueces? Rev Invest Clin 2000;52(2):203-210.

12. Panerai R, Peña J. Health Technology assessment methodologies for developing countries.W ashington,D.C., Pan American Health 0 rganization, 1990:1-33.

13. Fajardo-0 rtiz G .Atención médica. México, D.F.: Editorial la Prensa Médica Mexicana, 1983:552-554.

14. O rganización Panamericana de la Salud. El desarrollo de la evaluación de las tecnologías en salud en América Latina y El Caribe. Washington, D.C.: O PS 1998:1.1-1.2.

15. Instituto $\mathrm{N}$ acional de Estadística, Geografía e Informática. Anuario estadístico del estado de Morelos. Cuernavaca, Morelos, México:IN EGI, 1997. 16. Rodríguez-D omínguez J,Vandale-Toney S, López-Cervantes M. La utilización de nuevas técnicas médicas: la problemática para México. Gac Med Mex 1984;120(5):199-203.

17. Rodríguez-D omínguez J,Vandale-Toney S, D urán-A renas JLG, LópezMcnally AP, López-Cervantes M. Disponibilidad y utilización de innovaciones tecnológicas en atención médica en México. Bol 0 ficina Sanit Panam1984;97(4):283-285. 